\title{
MATthiola INCANA (BRASSICACEAE): PRIMER REgISTRO DE naturalización En ARgentina
}

\author{
MATTHIOLA INCANA (BRASSICACEAE): FIRST RECORD OF NATURALIZATION IN \\ Argentina
}

\author{
Adriel I. Jocou+i), Carlos R. Minué(i) y Ricardo Gandullo(i)
}

Departamento de Biología Aplicada. Facultad de Ciencias Agrarias, Universidad Nacional del Comahue, Río Negro, Argentina.

*adrieljocou@gmail.com

\section{Citar este artículo}

JOCOU, A. I., C. R. MINUÉ \& R. GANDULLO. 2019. Matthiola incana (Brassicaceae): primer registro de naturalización en Argentina. Bol. Soc. Argent. Bot. 53: 421-429.

DOI: http://dx.doi. org/10.31055/1851.2372.v54. n3.25366

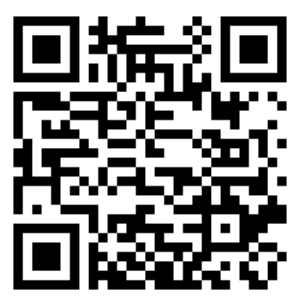

Recibido: 9 Mayo 2019

Aceptado: 21 Junio 2019

Publicado: 30 Septiembre 2019

Editora: Carolina I. Calviño (D)

\section{SUMMARY}

Background and aims: The genus Matthiola W. T. Aiton comprises between 50 and 60 species native to the Mediterranean, Central Asia and South Africa. M. incana (L.) W. T. Aiton, indigenous to southern Europe, is naturalized in other regions of the world. In Argentina it is cultivated as ornamental. From floristic observations, a naturalized population was found, which self-perpetuates without human intervention, on the coasts of the Patagonia Argentina. The objective of this work is to report for the first time the naturalization of $M$. incana in Argentina. M\&M: For the identification of the species, the classic methods of taxonomy were followed, specific bibliography was consulted and the collected material was contrasted with digital images of the lectotype. Seeds were collected from the naturalized population and the germinative power was evaluated through a germination test. The collected material that documents the present work was deposited in the ARC herbarium of the Universidad Nacional del Comahue.

Results: Matthiola incana is described. The common names and data on distribution, habitat, ecology and uses are included. Photographs are presented to complement the description. The germination test showed that $95 \%$ of the seeds germinated after 24 hours at $25^{\circ} \mathrm{C}$.

Conclusions: The naturalization of Matthiola incana is reported for the first time in Argentina, the only species of the genus in the country until now.

\section{KEY WORDS}

Brassicaceae, Flora Argentina, Matthiola, naturalization, new record, Patagonia.

\section{Resumen}

Introducción y objetivos: El género Matthiola W. T. Aiton posee entre 50 y 60 especies originarias del Mediterráneo, Asia central y Sudáfrica. M. incana (L.) W. T. Aiton, nativa del sur de Europa, se encuentra naturalizada en otras regiones del mundo. En Argentina es cultivada como ornamental. A partir de observaciones florísticas se halló una población naturalizada, que se autoperpetúa sin intervención del hombre, en las costas de la Patagonia Argentina. El objetivo de este trabajo es reportar por primera vez la naturalización de $M$. incana en Argentina.

M\&M: Para la identificación de la especie se siguieron los métodos clásicos de taxonomía, se consultó bibliografía específica y se contrastó el material recolectado con imágenes digitales del lectotipo. Se recolectaron semillas de la población naturalizada y se evaluó el poder germinativo a través de un ensayo de germinación. El material recolectado que documenta el presente trabajo fue depositado en el herbario ARC de la Universidad Nacional del Comahue.

Resultados: Se describe a Matthiola incana. Se incluyen nombres vulgares y datos sobre distribución, hábitat, ecología y usos. Se presenta una serie de fotografías de campo y bajo microscopio estereoscópico para complementar la descripción. El ensayo de germinación demostró que el 95\% de las semillas germina a las 24 hs a $25^{\circ} \mathrm{C}$.

Conclusiones: Se reporta por primera vez para Argentina la naturalización de Matthiola incana, única especie del género en el país hasta el momento.

Palabras clave

Brassicaceae, Flora Argentina, Matthiola, naturalización, nueva cita, Patagonia. 


\section{INTRODUCCIÓN}

El género Matthiola W. T. Aiton posee entre 50 y 60 especies originarias del Mediterráneo, Asia central y Sudáfrica (Dimitri, 1972; Al-Shehbaz, 2010; Flora of Pakistan, 2019). Algunas especies de este género son apreciadas como ornamentales en diferentes partes del mundo bajo el nombre vulgar de "alelí", en particular M. incana (L.) W. T. Aiton, por su llamativa floración y fragancia (Valdés, 1993; Al-Shehbaz, 2010; Flora of Pakistan, 2019).

Matthiola incana, originaria del sur de Europa, se encuentra naturalizada en el oeste de Europa y en la región Mediterránea, Australia, Chile, Norteamérica y Pakistán (Matthei et al., 1993; Valdés, 1993; Flora of Pakistan, 2019). En Argentina, tanto en territorio continental como en las Islas Malvinas, es muy frecuentemente cultivada por su uso ornamental (Spegazzini, 1905; Hicken, 1910; Dimitri, 1972; 1975). La especie fue reportada como escapada de cultivo por Spegazzini (1905) y Hicken (1910) sin evidencias de su naturalización, por lo que no se encuentra registrada para Argentina (Al-Shehbaz \& Salariato, 2012).

Esta contribución surge de diversas observaciones florísticas en las costas de la provincia de Río Negro. El objetivo de este trabajo es reportar por primera vez la naturalización de Matthiola incana en Argentina.

\section{Materiales y Métodos}

El material analizado se recolectó de una población de Matthiola incana ubicada en el Balneario Las Grutas, municipio de San Antonio Oeste, provincia de Río Negro (Fig. 1). Para su identificación se siguieron los métodos clásicos de taxonomía y se consultó bibliografía específica (Dimitri, 1972; Maire, 1976; Clemente Muñoz \& Pujadas Salvá, 1987; Valdés, 1993; Al-Shehbaz, 2010; Vizoso, 2011; Flora of Pakistan, 2019). Se contrastó el material con imágenes digitales del lectotipo (LINN-839.17) de The Linnean Society of London (http://linnean-online.org).

El estudio morfológico del material se realizó bajo microscopio estereoscópico y se tomaron fotografías para complementar la descripción.

Se recolectaron semillas de la población naturalizada y a los nueve días se evaluó el poder germinativo a través de un ensayo de germinación. Se utilizaron placas de Petri y sustrato inerte húmedo a temperatura ambiente $\left(25^{\circ} \mathrm{C}\right)$. Se evaluaron el porcentaje (\%) de germinación, a las 24 y $48 \mathrm{hs}$, de un total de 100 semillas, y el tiempo transcurrido hasta la aparición de los cotiledones.

El material recolectado que documenta el presente trabajo fue depositado en el herbario ARC de la Facultad de Ciencias Agrarias, Universidad Nacional del Comahue (código de herbario según Thiers, 2019).

\section{Resultados}

Matthiola incana (L.) W. T. Aiton, Hort. Kew., 2 ed. 4: 119. 1812. Cheiranthus incanus L., Sp. P1.: 662. 1753. TIPO: "Habitat in Hispaniae maritimis" (Lectotipo LINN-839.17 [foto]!, designado por Jafri, Fl. W. Pakistan 55: 200. 1973). Figs. 2-5.

Planta perenne de base leñosa, con indumento laxo de pelos ramificados cortos. Tallos erectos de hasta $70 \mathrm{~cm}$ de altura, tardíamente glabros. Hojas de hasta $14 \times 2,8 \mathrm{~cm}$, con abundantes pelos ramificados, glaucas, lineares, elípticas u oblongo-elípticas, en ocasiones levemente crespas, con margen entero, ápice obtuso, base atenuada. Pedicelos florales de 5-6 mm long., 10-20 × 1-2 mm en la fructificación. Flores de 20-25 mm diám. Sépalos ca. $12 \times 3 \mathrm{~mm}$, con abundantes pelos ramificados, estrechamente ovados, margen entero, ápice obtuso. Pétalos glabros; uña ca. $12 \mathrm{~mm}$ long., exerta, verde amarillenta; limbo de (9-)11-15 × 7-10 $\mathrm{mm}$, anchamente obovado u obcordado, patente, margen liso, completamente purpúreo, blanco o variegado. Androceo tetradínamo; anteras ca. 3 $\mathrm{mm}$ long., agudas; filamentos medianos (internos) ca. $6 \mathrm{~mm}$ long., ensanchados hacia la base, los laterales (externos) ca. $3 \mathrm{~mm}$ long., no ensanchados en la base. Nectarios 4 , ca. $0,7 \times 1 \mathrm{~mm}$, laterales, triangulares, verdes. Silicuas de (2,5-)5-10 × 0,3$0,5 \mathrm{~cm}$, erectas a patentes, con pelos ramificados cortos cuando inmaduras, color castaño claro cuando maduras, apéndice terminal de 0,7-2,5 mm long., truncado, con gibosidades menores a $1,5 \mathrm{~mm}$ long. Semillas 20-30 por lóculo, ca. $3 \mathrm{~mm}$ diám., suborbiculares, pardas, con ala de ca. $0,5 \mathrm{~mm}$ de ancho, de borde entero y sinuado, color crema. 


\section{A. I. Jocou et al. - Matthiola incana en Argentina}

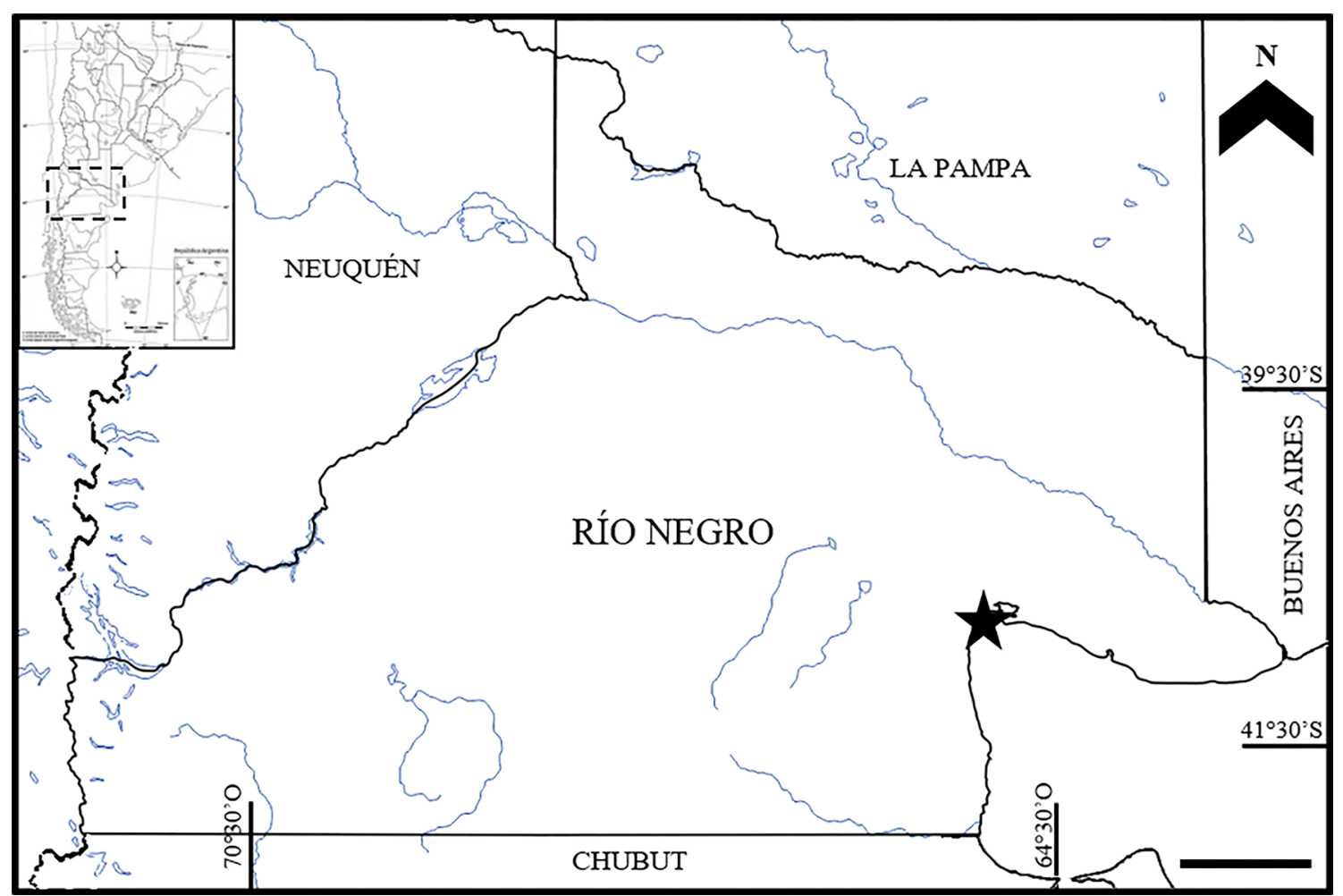

Fig. 1. Área de estudio. La estrella $(\star)$ indica la zona de hallazgo de la población naturalizada de Matthiola incana en el Balneario Las Grutas, provincia de Río Negro, Argentina. Escala $=80 \mathrm{~km}$.

Iconografia. Baxter (1843: fig. 445); Clemente Muñoz \& Pujadas Salvá (1987: 388); Kirtikar \& Basu (1918: fig. 60-B); Maire (1976: 8, fig. 3); Miller (1777: fig. 56); Mohlenbrock (1980: 265, fig. 125); Syme (1863: fig. 105).

Nombres vulgares. En Argentina se denomina vulgarmente a Matthiola incana como "alelí", "alhelí blanco" o "alelí común” (Spegazzini, 1905; Hicken, 1910; Dimitri, 1972).

Distribución, hábitat y ecología. Matthiola incana es nativa del sur de Europa, desde España a la antigua Yugoslavia, naturalizada en la región mediterránea y oeste de Europa (Valdés, 1993), Norteamérica, Australia (Rollins, 1981;Al-Shehbaz, 2010) y Chile (Matthei et al., 1993). Es frecuente en terrenos disturbados, suelos pedregosos, arenosos o calcáreos, áreas costeras y acantilados, lugares abandonados o en ruinas (Conti, 1900; Maire, 1976; Rollins, 1981; Valdés, 1993; Vizoso, 2011).
Matthiola incana fue reportada en Argentina en lugares urbanos por Hicken (1910) en escombros y terrenos disturbados cerca de San Isidro y en Lomas (Buenos Aires); y por Berg (1877), al mencionar que fue observada en una oportunidad en el barrio de La Boca (Buenos Aires). La población hallada crece a lo largo de los acantilados costeros del Balneario Las Grutas (Río Negro), sobre suelos pedregosos y arenosos, afectados por la acción del viento y el mar (Fig. 2). Se comprobó que esta especie es ampliamente cultivada en jardines de viviendas del área donde fue recolectada, por lo que la población naturalizada pudo iniciarse a partir de semillas de ejemplares cultivados. La alta producción de semillas aladas, fácilmente dispersadas por los fuertes vientos de la zona, favorece la invasión de otras áreas cercanas. En la zona de estudio, se observó la presencia de algunas plántulas de $M$. incana, con cotiledones orbiculares a suborbiculares de ápice obtuso y borde liso, glabros, de ca. 0,5 ×0,4 cm (Fig. 5D). 

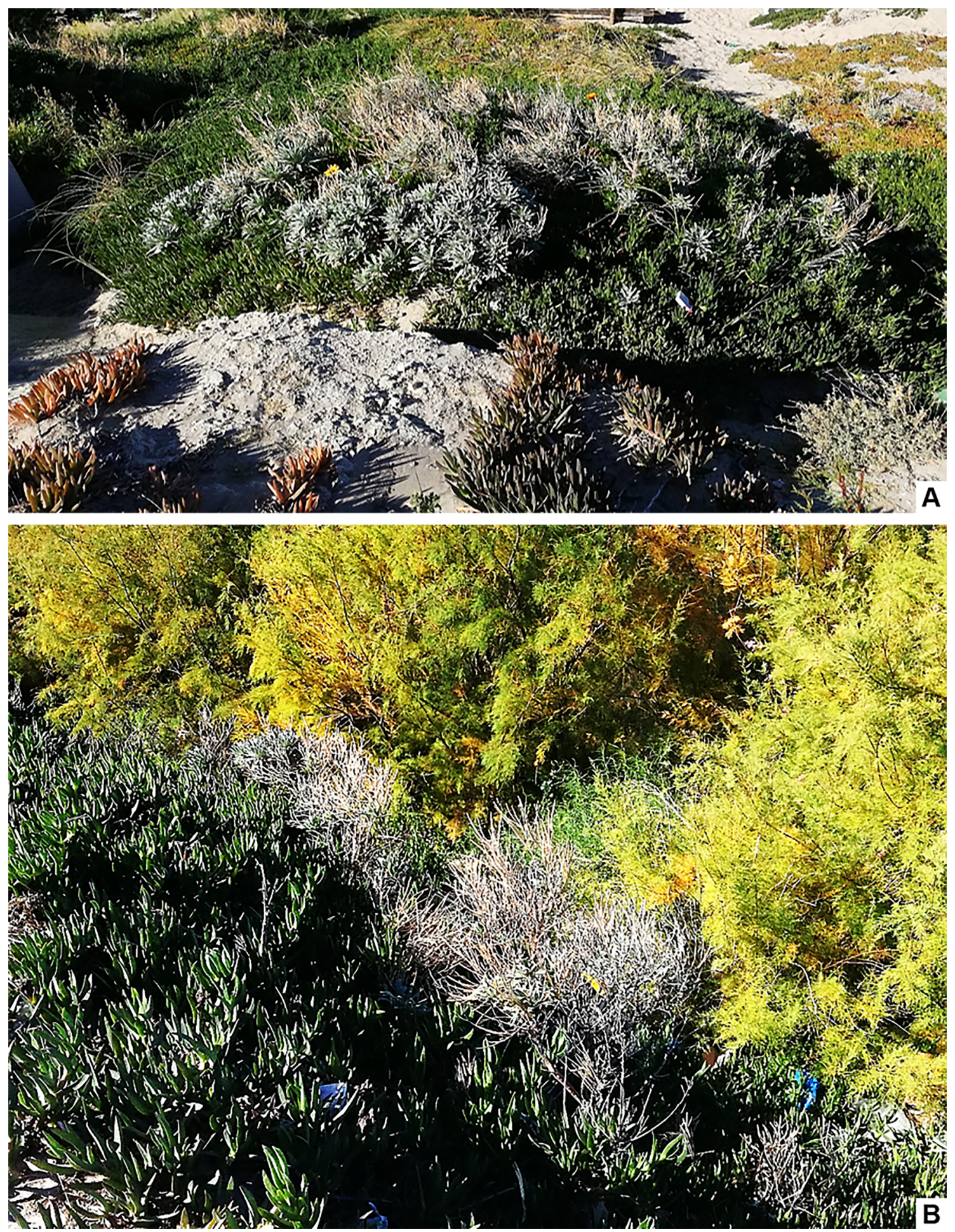

Fig. 2. Matthiola incana. A: Ejemplares en un acantilado, acompañados principalmente por Carpobrotus sp.

B: Ejemplares en la parte superior de un acantilado, acompañados principalmente por Tamarix ramossisima y Carpobrotus sp. 

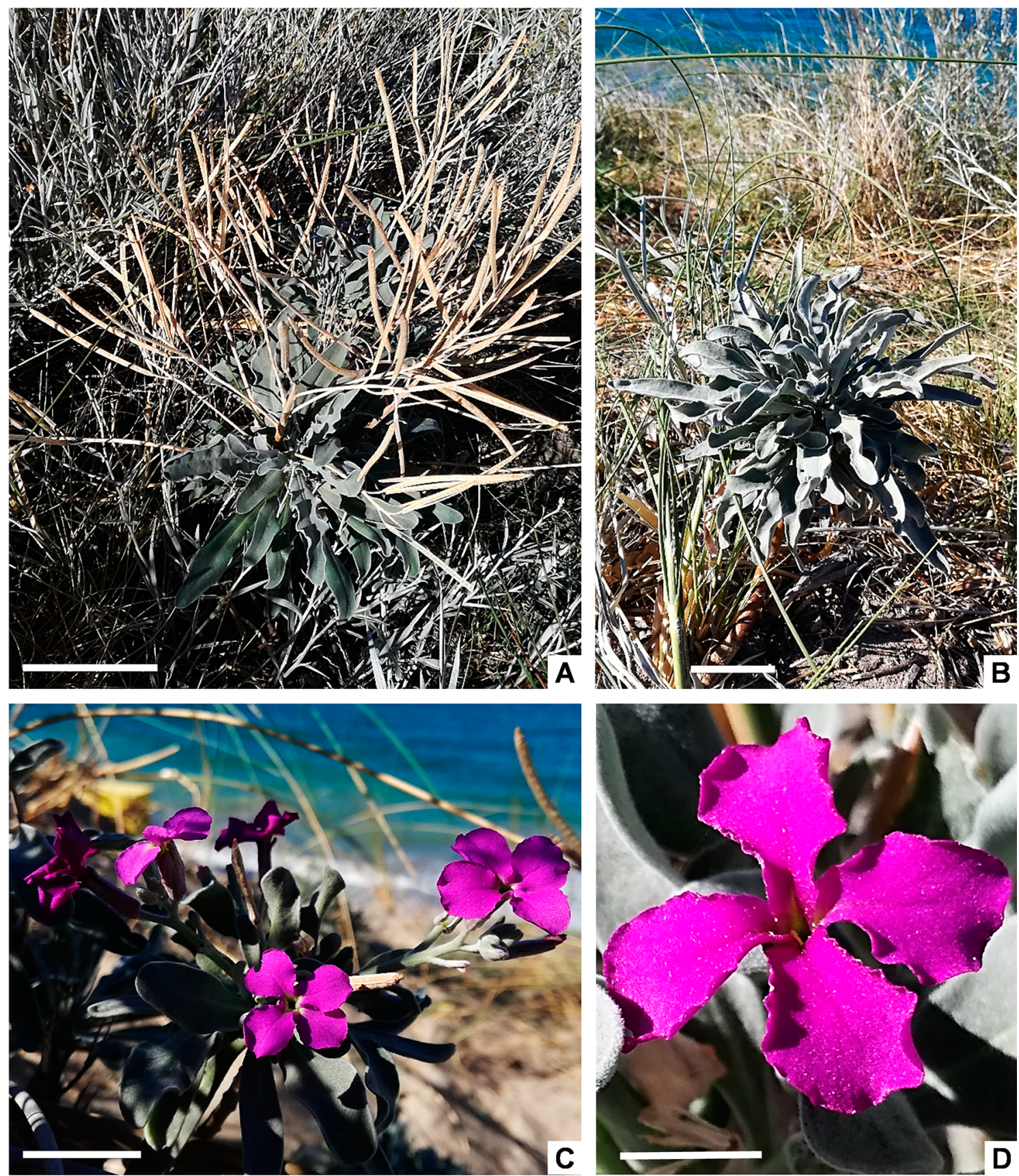

Fig. 3. Matthiola incana. A: Ejemplares en fructificación. B: Ejemplar en estado vegetativo, nótese las hojas dispuestas principalmente en roseta. C: Ejemplar en floración, nótese la coloración y dimensión de las flores. D: Flor. Escalas= A: $9 \mathrm{~cm}$; B: $5 \mathrm{~cm}$; $: 2,5 \mathrm{~cm}$; D: $1 \mathrm{~cm}$.

Se observó que cohabita con Carpobrotus sp. N. E. Br., Diplotaxis tenuifolia (L.) DC., Gazania rigens (L.) Gaertn., Grindelia chiloensis (Cornel.) Cabrera, Hyalis argentea D. Don ex Hook. \& Arn. var. latisquama Cabrera, Malva dendromorpha M.F. Ray, Mesembryanthemum nodiflorum L., Sporobolus rigens (Trin.) E. Desv. var. rigens, Suaeda argentinensis A. Soriano, S. divaricata 

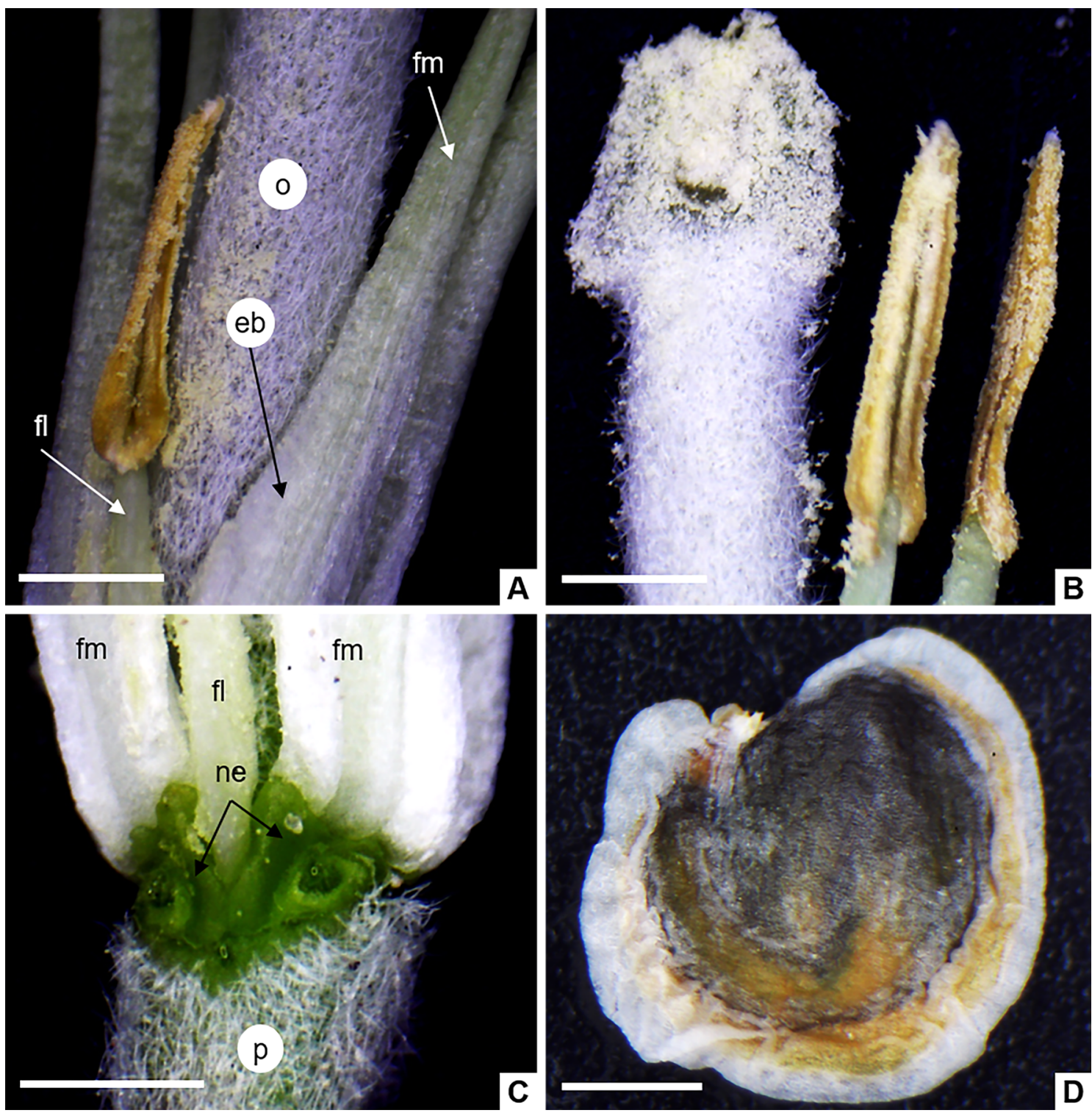

Fig. 4. Matthiola incana. A: Estambre lateral (externo), nótese la pubescencia del ovario y la base ensanchada de los filamentos medianos (internos). B: Ápice del gineceo y dos anteras de estambres medianos (internos). C: Nectarios triangulares. D: Semilla, nótese el ala alrededor de la misma. Abreviaturas= eb: ensanchamiento de la base; fl: filamento lateral; fm: filamento mediano; ne: nectarios; o: ovario; $p$ : pedicelo. Escalas= A-D: $1 \mathrm{~mm}$.

Moq. y Tamarix ramosissima Ledeb. De acuerdo a lo mencionado por Grieve et al. (2006) y Carter \& Grieve (2008) y debido a la presencia de especies indicadoras de suelos salinos, puede inferirse que $M$. incana presenta una tolerancia a niveles medios a altos de salinidad. El sitio de naturalización de $M$. incana presenta una temperatura máxima absoluta de $39,8^{\circ} \mathrm{C}$, una media de $14,4^{\circ} \mathrm{C}$, una mínima absoluta de $-7,3^{\circ} \mathrm{C}$ y una precipitación media anual de 189,9 mm (http://dpa.gov.ar/clima). En este sentido, puede afirmarse que se trata de una especie euriterma y tolerante a la sequía. 


\section{A. I. Jocou et al. - Matthiola incana en Argentina}
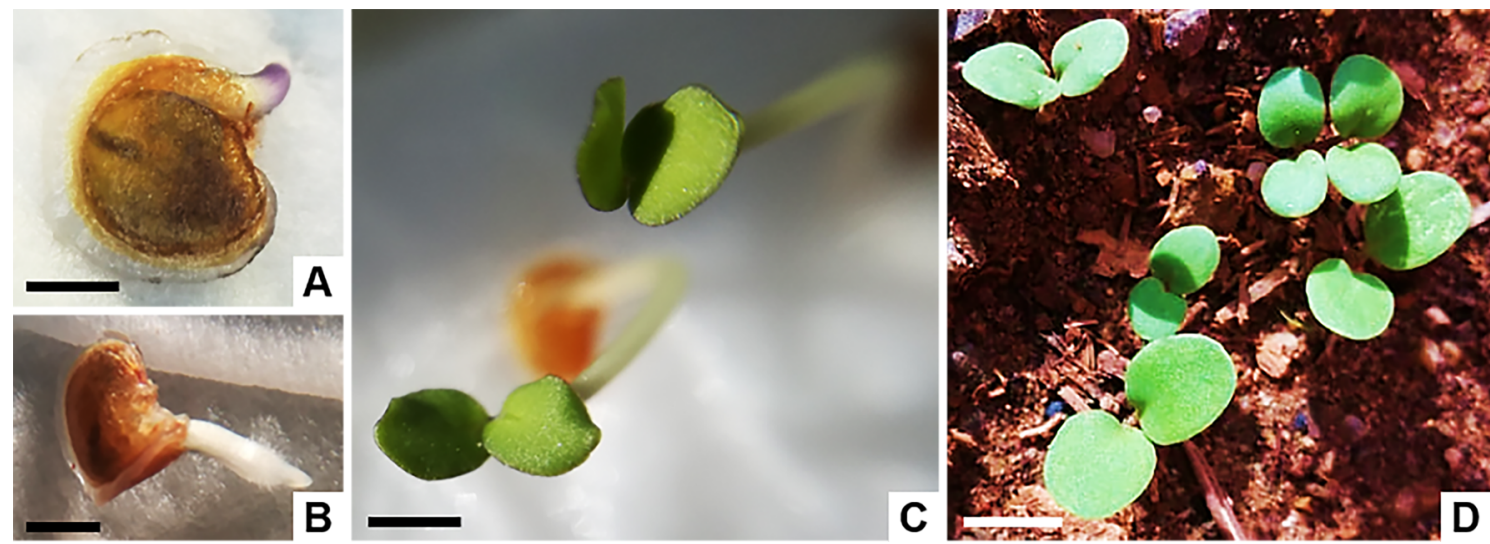

Fig. 5. Matthiola incana. A: Germinación de semillas a las 24 hs, nótese la hidratación del tegumento. B: Germinación a las 48 horas, nótense los pelos radicales. C: Cotiledones a las 72 hs. D: Plántulas, nótese la morfología de los cotiledones. Escalas= A-C: 1,5 mm; D: $4 \mathrm{~mm}$.

Usos. Se utiliza principalmente como ornamental por su vistosa y fragante floración (Dimitri, 1975; Cheers, 1999; Al-Shehbaz, 2010; Flora of Pakistan, 2019). Se destaca el uso de numerosos cultivares, entre ellos los de flores dobles, blancas, rosas, violetas, púrpuras o variegadas; con mayor o menor pubescencia y desde anuales a perennes (Dimitri, 1972; Maire, 1976; Cheers, 1999). Se cultivan principalmente para macizos o borduras y como flores de corte (Tlahuextl-Tlaxcalteca et al., 2005; Grieve et al., 2006; Eid et al., 2009). Cerón Martínez (2006) indica que en Ecuador es utilizada como medicinal para inflamaciones, tos y afecciones nerviosas, estomacales y cardíacas.

Observaciones. Para complementar la descripción y facilitar la interpretación de la misma, se presentan fotografias de ejemplares en fructificación (Fig. 3A), en estado vegetativo (Fig. 3B) y en floración (Fig. 3CD). Además, se detallan estructuras internas de la flor (Fig. 4A-C) y la semilla (Fig. 4D).

Las características que permiten distinguir al género Matthiola son la presencia de silicua dehiscente de sección circular a tetragonal, con el nervio medio bien marcado y semillas uniseriadas comprimidas, surborbiculares, con ala periférica completa. Por otra parte, $M$. incana se distingue fácilmente de otras especies por los pedicelos fructíferos mayores a $8 \mathrm{~mm}$ long., silicuas entre 3-5 mm de ancho, sin glándulas estipitadas, pétalos con limbo de 11-15 × 7-10 $\mathrm{mm}$ y hojas de margen entero.
El ensayo de germinación demostró que el $95 \%$ de las semillas germinaron a las $24 \mathrm{hs}$ y a las $72 \mathrm{hs}$ las 100 semillas alcanzaron el estado de plántula (Fig. 5A-C), con un completo desarrollo de los cotiledones. Esta característica, junto a la alta producción de semillas aladas, son de relevancia en el proceso de naturalización. Asimismo, la población en el área de estudio se encuentra establecida, sin intervención del hombre, desde al menos ocho años (comunicación personal con pobladores). Estos datos confirman que Matthiola incana se encuentra naturalizada, de acuerdo a los conceptos establecidos por Pyšek et al. (2004).

Material estudiado. ARGENTINA. Prov. Río Negro: Dpto. San Antonio, San Antonio Oeste, Balneario Las Grutas, 21-IV-2019, Jocou \& Minué 2239 (ARC).

\section{Contribución DE los AUTORES}

Todos los autores participaron conjuntamente y a partes iguales tanto en la recolección de datos como en la redacción del manuscrito. El material vegetal fue recolectado por Adriel Jocou y Carlos Minué.

\section{Agradecimientos}

A los revisores anónimos y la editora Carolina Calviño, por sus valiosos comentarios y sugerencias 
que permitieron mejorar el manuscrito. $\mathrm{Al} \mathrm{Sr}$. Graciano, de la Biblioteca Digital del Real Jardín Botánico de Madrid por proveer parte de la bibliografía solicitada.

\section{Bibliografía}

AL-SHEHBAZ, I. A. \& D. L. SALARIATO. 2012. Flora argentina: flora vascular de la República Argentina. Diotyledoneae, Brassicaceae, vol. 8. Editorial Sigma, Buenos Aires.

AL-SHEHBAZ, I. A. 2010. Matthiola. In: Flora of North America Editorial Committee (eds.), Flora of North America, vol. 7, pp. 253-255. Oxford University Press, New York. Disponible on-line en: eFloras.org.

BAXTER, W. 1843. British Phaenogamous Botany; or, Figures and descriptions of the genera of British flowering plants, vol. 6. Published by the Author, London. Disponible en:

https://www.biodiversitylibrary.org/item/192133

BERG, C. 1877. Enumeración de las plantas europeas que se hallan como silvestres en la provincia de Buenos Aires y en Patagonia. Anales Soc. Ci. Argent. 3: 183-206. https://doi.org/10.5962/bhl.title.9301

CARTER, C. T. \& C. M GRIEVE. 2008. Salt tolerance of floriculture crops. In: KHAN, M. A. \& D. J. WEBER (eds.), Ecophysiology of high salinity tolerant plants, Tasks for vegetation science, vol. 40, pp. 279288. Springer, Dordrecht. https://doi.org/10.1007/1-4020-4018-0_19

CERÓN MARTÍNEZ, C. E. 2006. Plantas medicinales de los Andes ecuatorianos. En: MORAES, M., B. ØLLGAARD, L. P. KVIST, F. BORCHSENIUS \& H. BALSLEV (eds.), Botánica Económica de los Andes Centrales, pp. 285-293. Universidad Mayor de San Andrés, La Paz.

CHEERS, G. 1999. Botanica, the illustrated A-Z of over 10,000 garden plants and how to cultivate them, 3ra ed. Könemann, Milsons Point.

CLEMENTE MUÑOZ, M. \& A. PUJADAS SALVÁ. 1987. Matthiola. En: VALDÉS, B., S. TALAVERA \& E. FERNÁNDEZ-GALIANO (eds.), Flora de Andalucía Occidental, vol. 1, pp. 86-97. Ketres editora, Barcelona.

CONTI, P. 1900. Les espèces du genre Matthiola. Mém. Herb. Boissier 18A:1-118.

DIMITRI, M. J. 1972. Matthiola. En: DIMITRI, M. J. (dir.), Enciclopedia Argentina de Agricultura y Jardinería, 2da ed., vol. 1, pp. 416. ACME, Buenos Aires.
DIMITRI, M. J. 1975. Consideraciones sobre la vegetación espontánea y las plantas cultivadas en las Islas Malvinas. Anales Soc. Ci. Argent. 199: 99-132.

EID, A. R., M. N. AWAD \& H. A. HAMOUDA. 2009.

Evaluate effectiveness of bio and mineral fertilization on the growth parameters and marketable cut flowers of Matthiola incana L. American-Eurasian J. Agric. \& Environ. Sci. 5: 509-518.

FLORA OF PAKISTAN, 2019. Matthiola. Disponible en: http://www.tropicos.org/Project/Pakistan [Consulta: 29-IV-2019].

GRIEVE, C. M., J. A. POSS \& C. AMRHEIN. 2006. Response of Matthiola incana to irrigation with saline wastewaters. HortScience 41: 119-123. https://doi.org/10.21273/HORTSCI.41.1.119

HICKEN, C. M. 1910. Chloris platensis Argentina. Apuntes Hist. Nat. 2. https://doi.org/10.5962/bhl.title.8586

KIRTIKAR, K. R. \& B. D. BASU. 1918. Indian medicinal plants. Indian press, Allahabad. https://doi.org/10.5962/bhl.title.137025

MAIRE, R. 1976. Flore de L'Afrique du Nord (Maroc, Algérie, Tunisie, Tripolitaine, Cyrénaïque et Sahara), vol. 14. Lechevalier, Paris.

MATTHEI, O., C. MARTICORENA \& T. F. STUESSY. 1993. La flora adventicia del Archipiélago de Juan Fernández. Gayana Bot. 50: 69-102.

MILLER, J. 1777. An illustration of the sexual system of Linnaeus. Published and sold by the Author, London.

MOHLENBROCK, R. H. 1980. The Illustrated Flora of Illinois. Flowering Plants: Willows to Mustards. Southern Illinois University Press, Carbondale.

PYŠEK, P., D. M. RICHARDSON, M. REJMÁNEK, G. L. WEBSTER, M. WILLIAMSON \& J. KIRSCHNER. 2004. Alien plants in checklists and floras: towards better communication between taxonomists and ecologists. Taxon 53: 131-143. https://doi.org/10.2307/4135498

ROLLINS, R. C. 1981. Weeds of the Cruciferae (Brassicaceae) in North America. J. Arnold Arbor. 62: 517-540.

SPEGAZZINI, C. 1905. Flora de la Provincia de Buenos Aires, vol. 1. M. Biedma e Hijo, Buenos Aires. https://doi.org/10.5962/bhl.title.9303

SYME, J. T. B. 1863. English botany; or, Coloured figures of British plants, 3ra. ed, vol. 1. Robert Hardwicke, London. Disponible en: https://www. biodiversitylibrary.org/item/45036

THIERS, B. 2019. Index Herbariorum: A global directory of public herbaria and associated staff. 


\section{A. I. Jocou et al. - Matthiola incana en Argentina}

New York Botanical Garden's Virtual Herbarium. Disponible en: http://sweetgum.nybg.org/science/ ih [Acceso: abril 2019].

TLAHUEXTL-TLAXCALTECA, C., J. M. ÁVILASÁNCHEZ \& H. LESZCZYÑSKA-BORYS. 2005.

Flores de corte y follaje en florerías y mercados de Puebla, México. Revista Chapingo, Ser. Hort. 11:323327. https://doi.org/10.5154/r.rchsh.2004.04.026

VALDÉS, B. 1993. Matthiola. En: CASTROVIEJO, S., C. AEDO, C. GÓMEZ CAMPO, M. LAÍNZ,
P. MONTSERRAT, R. MORALES, F. MUÑOZ GARMENDIA, G. NIETO FELINER, E. RICO, S. TALAVERA \& L. VILLAR (eds.). Flora Ibérica: Plantas vasculares de la Peninsula Iberica e Islas Baleares, vol. 4, pp. 86-97. CSIC, Madrid.

VIZOSO, M. T. 2011. Matthiola. En BLANCA G., B. CABEZUDO, M. CUETO, C. SALAZAR \& C. MORALES TORRES (eds.), Flora Vascular de Andalucía Oriental, 2da ed.,pp. 946-948. Universidades de Almería, Granada, Jaén y Málaga, Granada. 
